\title{
Cognición implícita: un factor para considerar en la educación médica
}

\author{
Implicit cognition: a factor to consider in medical education
}

\author{
Est. Mariana Ríos ${ }^{a}$ Dr. Eduardo Durante ${ }^{b}$, Dr. Alfredo Eymann ${ }^{c}$ y Dr. Pablo Argibay ${ }^{a}$
}

\section{RESUMEN}

La existencia de prejuicios, a menudo inconscientes, es una limitación para la empatía médica y la relación médico-paciente.

El término cognición implícita se refiere a las influencias inconscientes (conocimiento previo, percepción y memoria) que, al influir en el comportamiento de las personas, afecta su desempeño o la propia toma de decisiones. A través del aprendizaje, asimilamos la información que nos brinda el medio y nos apropiamos del conocimiento considerando sus distintas dimensiones (conceptos, procedimientos, actitudes y valores). Los mecanismos de aprendizaje implícito son lentos, no suelen ser percibidos conscientemente por las personas y requieren la asociación de los estímulos secuenciales y del almacenamiento de la información concerniente a las relaciones predictivas entresucesos. Durante el aprendizaje y luego en el ejercicio de la medicina en particular, la existencia de actitudes implícitas negativas relacionadas con el género o las etnias, por ejemplo, podría limitar no solo la adquisición de conocimientos y sus habilidades académicas, sino también el desarrollo de la empatía necesaria durante la atención de los pacientes. La identificación de estas a través del test de asociaciones implícitas (TAI) permitiría caracterizar o predecir las dificultades de aprendizaje a nivel emocional y relacional de los estudiantes. Eso podría ser relevante en la modificación de los planes de estudio para que reciba, durante su formación, las herramientas necesarias para lograr un desempeño adecuado. Este artículo pretende introducir en el campo de la medicina el tema de la cognición implícita asociado a la educación médica.

Palabras clave: aprendizaje, cognición, educación médica, prejuicios y asociación implícita.

http:/ /dx.doi.org/10.5546/aap.2014.59

\section{Cognición implícita}

El término cognición implícita se refiere a las influencias inconscientes, como el conocimiento previo, la percepción o la memoria, que influyen en el comportamiento de una persona, a pesar de que esa persona no tiene conciencia alguna de esas influencias. ${ }^{1}$ Las teorías conductistas aplicadas al aprendizaje están más preocupadas por las respuestas explícitas de las personas a las estrategias de educación que a los procesos mentales, cognitivos o neurobiológicos utilizados para aprender y resolver problemas. Se suele preguntar al estudiante de qué manera cree que ha aprendido tal o cual habilidad, infiriendo de esa narración consciente, potenciales mecanismos de aprendizaje. Si bien estos esquemas pueden resultar útiles, es indudable que la enseñanza de una persona en cualquier dominio, pero fundamentalmente en la toma de decisiones que afectan a otros, debe incluir un profundo conocimiento de hábitos y prejuicios acuñados inconscientemente, en muchos casos en etapas bastante tempranas del desarrollo. Independientemente de la denominada "memoria implícita", en la cual las experiencias anteriores ayudan en el desempeño de una tarea sin ser consciente la persona de estas experiencias previas, ${ }^{2}$ la cognición implícita incorpora influencias negativas (prejuicios) que afectan la toma de decisiones.

La cognición requiere, entre otras cosas, representaciones implícitas y explícitas en distintos dominios. Una representación mental (o representación cognitiva), en filosofía de la mente, psicología cognitiva, neurociencia o ciencia cognitiva en general, es un símbolo interno e hipotético que representa la realidad exterior, o bien un proceso mental que utiliza un símbolo. En otras palabras, es un sistema formal específico que permite hacer explícitas ciertas entidades o tipos de información. ${ }^{3}$

\section{Procesos explícitos}

Para Karmiloff-Smith (1992), citado por Pozo, ${ }^{4}$ la función de los procesos explícitos sería posiblemente la de 
favorecer la redescripción representacional de aquellos productos de la cognición implícita destinados a facilitar el acceso al propio sistema cognitivo y a las relaciones entre representaciones generando nuevas formas de conocimiento. Según Donald (1991), citado por Pozo, ${ }^{4}$ la conversión de las representaciones de conocimiento mediante su explicitación progresiva reduciría la dependencia de la persona con respecto al ambiente, permitiendo su conocimiento o nuevas formas de organizarlas independientemente de la estructura que los estímulos adoptaran en el mundo. Por lo tanto, la función de las representaciones explícitas sería permitir el conocimiento del mundo de la persona a través de la acción sobre las propias representaciones que tiene sobre el mundo.

El aprendizaje explícito, utilizado durante la resolución de problemas constituidos por series de ordenamientos con elementos faltantes o en la búsqueda de las diferencias entre dos figuras, suele ser rápido. Puede ocurrir tras el primer esfuerzo, implica la asociación de estímulos simultáneos y permite almacenar información acerca de un suceso que se da en un mismo tiempo y lugar determinados produciendo una sensación de familiaridad con sucesos previos. ${ }^{5}$

\section{Procesos implícitos}

Los elementos cognitivos implícitos, que operan libres del control o del conocimiento de la persona, actúan arbitrariamente sobre la percepción, la codificación, la interpretación de la información y las conductas resultantes. Al ser implícitos, no se basan en teorías formales y el impacto causado sobre el comportamiento de las personas no es percibido necesariamente de manera consciente, ${ }^{6}$ es decir, que estos procesos actúan como automáticos al iniciarse con la exposición a determinados estímulos. Para Moskowitz (2005), citado por Dorantes Argandar, ${ }^{7}$ un proceso implícito se considera automático si: 1) no es intencional (se inicia inconscientemente); 2) es imposible de controlar (la decisión de no activar el proceso no detiene su ocurrencia); 3) es extremadamente eficiente (ocurre independientemente de otras actividades simultáneas); 4) procede sin que nos percatemos de ello.

El aprendizaje implícito es lento, permite acumular destreza a través de reiterados ensayos, requiere la asociación de estímulos secuenciales y almacena información concerniente a las relaciones predictivas entre sucesos. La persona dice no ser consciente del contenido del aprendizaje. $^{8}$
Un mecanismo de aprendizaje implícito (por ejemplo, la adquisición del lenguaje materno, aprender a andar en bicicleta o a nadar) puede generar representaciones que controlen la conducta y ser un desencadenante del aprendizaje explícito. ${ }^{5}$

Una forma de conocimiento implícito particular, si no innato, al menos enraizado en la evolución del homo sapiens, es la denominada teoría de la mente ("ToM"). Se dice que un individuo tiene una "ToM" si es capaz de imputar estados mentales a sí mismo y a los demás. Este sistema de inferencias es visto como una teoría porque tales estados no son directamente observables, y puede utilizarse para predecir acerca de los comportamiento de los demás. ${ }^{9} \mathrm{La}$ "ToM" sería la facultad para deducir el estado mental inobservable que está causando una conducta previamente observada, se relaciona con los deseos, las intenciones y las creencias del otro, y es un mecanismo heredado genéticamente e implícito. Se desarrolla entre los 2 y los 5 años de edad, ${ }^{10}$ está afectada en los niños ${ }^{9}$ es independiente del coeficiente intelectual. Por otra parte, se ha sostenido una forma de cognición implícita bajo la forma del teorema de Bayes. ${ }^{11}$ El bayesianismo implícito supone que una persona actualizará sus creencias acerca de los demás sobre la base de nueva información. Si bien dichas actitudes se pueden concientizar metacognitivamente, su uso supone mecanismos implícitos. La persona no es conscientemente un evaluador de situaciones con una calculadora en la mano para analizar la probabilidad previa y la probabilidad posprueba. Ni siquiera es factible que las personas en general actúen con cierta noción bayesiana ingenua. ${ }^{12}$ Lo que se propone es que durante la toma de decisiones, los agentes sociales actúan implícitamente, como si fueran analistas bayesianos, de la misma manera en que las hormigas construyen sus hormigueros como si siguieran cierta arquitectura como lo realizado por Carlos Páez Vilaró (constructor uruguayo). Dichos modelos (Bayes, Páez Vilaró) de alguna manera describen lo que hacen los agentes (humanos, hormigas), aunque estos no sean conscientes de ello.

\section{Teorías implícitas}

Las "teorías implícitas" ("TIs"), importantes en la enseñanza y en el aprendizaje en todos los niveles, constituyen para $\mathrm{Pozo}^{4}$ un tercer nivel en el análisis de las representaciones mentales. Son un conjunto de creencias que una persona posee respecto de cómo son las personas, la naturaleza humana o los grupos sociales. 
El concepto de "TIs" no se refiere a la inferencia de estados mentales en el otro ("ToM"), sino a la asignación de una cualidad o calificativo hacia el otro. Estos conocimientos se organizan de modo más o menos coherente dentro de las "Tls" de cada persona y establecen relaciones entre los diferentes aspectos de la realidad..$^{13} \mathrm{Al}$ igual que los esquemas, las "TIs" cumplen un papel relevante en los procesos de comprensión, memoria y planificación de acciones. Son adquiridas desde edades muy tempranas a partir de las experiencias de cada persona en situaciones recurrentes propiciadas por las normas sociales y culturales. Hirschfeld (1995), citado por Estrada, Oyarzún y Yzerbyt, ${ }^{13}$ plantea la importancia del contexto relacional instalado en un sistema cognitivo innato que le permite a una persona clasificar a los seres humanos utilizando diferencias superficiales observables determinadas por las representaciones culturales sobre una categoría (etnia, peso, edad, etc.) que ha reforzado el ambiente del niño y no el sistema cognitivo innato. Dentro de las funciones de las "TIs", podemos destacar que permiten: 1) comprender y simplificar la realidad social; 2) organizar la realidad social "etiquetando" a las observaciones y experiencias vividas cotidianamente; 3 ) compartir el "sentido común" que reúne y sintetiza nuestros valores y formas de interpretar la realidad y, 4) proteger nuestro autoconcepto y el de nuestro grupo adoptando un marco justificado para las actitudes intergrupo y las desigualdades sociales. ${ }^{14}$

Las "Tls" se agrupan en tres grandes grupos: las "Tls" sobre la personalidad, relacionadas con el conocimiento y la forma en que utilizamos ese conocimiento para inferir la personalidad de los demás; las "Tls" respecto de la naturaleza humana, creencias generales que cada persona posee sobre el ser humano (incluyen las teorías sobre el locus de control y las teorías sobre la naturaleza humana propiamente dicha) y, por último, las "Tls" que hacen referencia a la naturaleza u origen de los grupos, que reagrupan nuestras creencias y conocimientos en relación con las condiciones de pertenencia de una persona a un grupo social dado.

\section{Esencialismo psicológico}

Scherif (1948), citado por Estrada, Oyarzún y Eyzerbyt, ${ }^{13}$ observó que algunas personas describen los acontecimientos sociales en términos de "esencia", es decir, incluyendo las cualidades inherentes a un grupo.
"La esencia puede ser definida como un conjunto de características y propiedades biológicas o socioculturales instaladas en la base de un grupo social ... que constituye el núcleo estable compartido por los miembros de un mismo grupo, siendo ella la condición necesaria y suficiente para pertenecer a una categoría social". ${ }^{13} \mathrm{El}$ término "esencialismo psicológico", utilizado por primera vez por Allport, puede definirse como la tendencia a creer que en la base de la existencia de un grupo social hay una esencia compartida por todos sus miembros. ${ }^{15,16}$ Puede considerarse como una teoría implícita relacionada con la creencia grupal de la existencia de una esencia que le permite a los miembros de un grupo reconocer en él componentes similares o comunes a todos los miembros. Para Medin, ${ }_{1}^{17}$ la similitud perceptiva se basa en representaciones de características de superficie (observables) que son conectadas a características profundas (no observables a simple vista).

Dentro de las consecuecias del esencialismo psicológico se destaca la inalterabilidad y el poder inductivo de la categoría. Es decir, que la mera pertenencia de un sujeto a un grupo es vista como inmutable (inalterabilidad) y el solo hecho de saber a qué grupo pertenece permite inferir nuevas características no observadas (poder inductivo de la categoría). Para Yzerbyt y Schadron (1996), citado por Estrada Goic, ${ }^{18}$ según el "síndrome esencialista", un grupo o categoría social se encuentra "esencializado" cuando: a) es percibido como una entidad (grupo visto como homogéneo); b) la pertenencia categorial es inalterable, inductiva, dotada de significación profunda, y excluyente (si se pertenece a una cierta categoría social, mapuche, por ejemplo, no se puede dejar de pertenecer a ella y nos permitirá predecir características comunes a sus miembros); c) los atributos de la categoría tienen fuertes conexiones entre sí.

Estudios recientes sobre el efecto del esencialismo sobre la explicación de las diferencias grupales explican que el esencialismo modula el uso de los factores explicativos biológicos y culturales para los intergrupos y no para los intragrupos, ${ }^{19}$ por lo tanto, a la hora de explicar las diferencias intergrupo, las personas se basan en factores biológicos (internos) y sociales (externo o culturales).

Otros estudios revelan el fenómeno de la infrahumanización en relación con el esencialismo y el intragrupo. ${ }^{6}$ Para estos autores, como las personas tienden al egocentrismo asignan al intragrupo, es decir, al grupo al cual pertenecen, 
su propia esencia humana considerada superior, y dotan al intergrupo de una esencia menos humana e inferior, dando lugar al fenómeno de infrahumanización. Bar-Tal ${ }^{20}$ propuso que este fenómeno nos puede conducir a la percepción de una persona o grupo social como ilegítimo (o inferior), y provocar emociones negativas y de rechazo - producto de esa falta de humanidadhacia ellos. Otro aspecto importante que se ha investigado es el efecto del esencialismo sobre los estereotipos y los prejuicios. Gordon Allport (1954), citado por Banaji y Bhaskar ${ }^{21}$ en su tratado La naturaleza del prejuicio, postuló que la creencia en la existencia de esencias en los grupos es uno de los aspectos de la percepción social ligada directamente al prejuicio. También afirmó que los conceptos de actitud (prejuicios) y creencias (estereotipos) están profundamente arraigados en las formas corrientes en las que los seres humanos percibimos, categorizamos y recordamos.

\section{Actitudes}

Una actitud es una asociación entre un concepto y una evaluación (positiva-negativa o favorable-desfavorable) que nos va a permitir guiar nuestro juicio, pensamiento o comportamiento.

Los psicólogos Anthony Greenwald y Banaji Mahzarin definen las actitudes implícitas como "las huellas de la experiencia pasada introspectivamente, no identificada o erróneamente identificada que median un sentimiento favorable o desfavorable, el pensamiento o la acción hacia un objeto social". ${ }^{22} \mathrm{Al}$ hablar de "sentimiento favorable o desfavorable" hacia un objeto, los autores aluden al vínculo de la asociación entre las evaluaciones y los conceptos; la frase "introspectivamente no identificada" se relaciona con la idea de la existencia de actitudes implícitas por fuera de la conciencia; "erróneamente identificada" alude a la diferencia entre las actitudes explícitas (experimentadas conscientemente como sentimientos) y las implícitas (experimentadas inconscientemente) y, por último, "las huellas de la experiencia pasada" hace referencia a la idea de que las actitudes implícitas estarían determinadas por la acumulación de las experiencias vividas.

Las actitudes explícitas reflejan los valores, las creencias conscientes, las respuestas deseadas y tienden a predecir las conductas deliberadas que son bastante fáciles de controlar. En cambio, las actitudes implícitas, además de reflejar la experiencia, tienden a predecir comportamientos que son más espontáneos y difíciles de controlar. Por ejemplo, durante la consulta de un anciano, un médico puede conscientemente estar en desacuerdo con las ideas negativas y mantener una actitud positiva explícita hacia los ancianos y el envejecimiento. Pero implícitamente esta información negativa puede almacenarse como una asociación entre negatividad y vejez. Por lo tanto, la educación médica en este aspecto es relevante porque al centrarse en el estudiante estimula la explicitación de estas actitudes, solo así esta última asociación se modificará; de lo contrario, permanecerá de manera inconsciente en la persona.

No existe una relación entre nuestras expresiones implícitas de las creencias y las actitudes con las versiones explícitas de estas. Por lo tanto, es importante entender la naturaleza de las relaciones y las consecuencias de esas disociaciones. También es necesario entender que hay consecuencias de las expresiones implícitas y de las creencias que no han sido atendidas por el poder de recompensa y castigo basado en la membresía del intragrupo al que se pertenece. ${ }^{21}$

Los seres humanos dividen el mundo en categorías para poder atribuirle un sentido y los grupos son las categorías utilizadas al referenciar a las personas. Moskowitz (2005), citado por Dorantes Argandar, ${ }^{7}$ afirma que una de las razones por las que no estamos conscientes de muchas influencias y prejuicios en nuestros juicios es porque estos ocurren fuera de la conciencia. Como el procesamiento es automático, su influencia en el juicio y en la toma de decisiones es difícil de eliminar y corregir completamente. ${ }^{23}$

\section{Esquemas mentales}

Para la psicología cognitiva, un esquema: 1) es un conjunto de conocimiento interactivo con la información entrante; 2) tiene una estructura interna consistente encargada de organizar, en forma particular, la información entrante; 3) se encarga de englobar la información genérica prototípica e impone una estructura procesando los casos específicos según el prototipo esquemático; y 4) a partir de la activación de una parte producirá la activación de la totalidad.

Las personas utilizan los "esquemas" para percibir, codificar y recuperar la información. Estos cumplen funciones adaptativas al facilitar la eficacia del procesamiento de la información, influyen en la evocación de la memoria y de los juicios, y sirven como una fuente de claves. También pueden servir como una guía para 
adivinar y reconstruir cuando los intentos de evocación fallan o producen resultados ambiguos. ${ }^{24}$ Por ejemplo, durante un episodio de internación hospitalaria un médico expuesto previamente a una experiencia desagradable con un miembro de un intergrupo extraño (nacionalidad, clases sociales, etc.) podrá estar influido inconscientente de forma negativa hacia los sentimientos, los pensamientos o la forma de actuar del paciente. Estas creencias mantenidas por una persona en relación con un grupo social reciben el nombre de "estereotipos" $\mathrm{y}$, al activarse, sesgan no solo el pensamiento y el recuerdo, sino también el comportamiento de las personas. Según Schneider (2004), citado por Dorantes Argandar, ${ }^{7}$ muchas formas de discriminación son bastante sutiles y pueden resultar de prejuicios implícitos, sesgos inconscientes, presiones sociales o del simple descuido.

\section{Test de asociación implícita}

Es un instrumento creado por los integrantes de Project Implicit, ${ }^{25}$ Banaji, Greenwald y Nosek, para medir las actitudes implícitas evaluando las preferencias hacia un intergrupo (etnia o género, etc.). Está disponible en línea (http:// implicit.harvard.edu/implicit/) e infiere, a partir de la relación entre un estímulo presentado y la velocidad con que se responde a una tarea específica, las asociaciones que conscientemente una persona puede detectar apareando dos tareas (juicio de un rostro y del valor de una palabra) en un único par de respuestas. Para Moskowitz (2005), citado por Dorantes Argandar, ${ }^{7}$ aparear dos tareas en una respuesta significa que la persona deberá tomar dos decisiones diferentes al realizar exactamente la misma cosa.

En este test, los participantes comienzan practicando la clasificación de estímulos de grupos sociales en una de dos categorías: por ejemplo, categorizando rostros de personas jóvenes y viejas, cada uno asignado a una tecla de la computadora particular. Luego practican la clasificación de adjetivos "positivos y negativos" usando las mismas teclas de respuesta. Una vez iniciado el test, en el primer bloque realizan ambas tareas de clasificación simultáneamente, es decir, que alternan rostros de personas jóvenes y viejas con adjetivos "positivos y negativos". Esta tarea implica que un grupo social y una categoría evaluativa compartan una tecla de respuesta ( $1^{a}$ tecla: rostros de personas jóvenes y palabras positivas. $2^{a}$ tecla: rostros de personas viejas y palabras negativas) y al reflejar el valor estereotípico asumido de estas categorías sociales se considera una condición compatible. Posteriormente, las etiquetas de categoría social son revertidas, creándose así la condición incompatible. Por lo tanto, se crea un conflicto entre las asociaciones estereotípicas relacionadas con el grupo social y los adjetivos que necesitan ser categorizados con la misma tecla. Este conflicto da lugar a una interferencia cognitiva que se traduce en el incremento de los tiempos de reacción ${ }^{26}$ y provoca en la persona, al permitirle enfrentarse con su propia realidad inconsciente, una incompatibilidad entre sus actitudes inconscientes y los valores que cree sostener.

Es importante destacar que, al igual que los procesos y las teorías descritas anteriormente, la personalidad se va desarrollando a edades tempranas, producto de la continua interacción con los otros y con las sucesivas situaciones sociales. La manifestación de la personalidad está representada por las actitudes emocionales e intelectuales. Por lo tanto, como todo mecanismo de aprendizaje implícito puede generar representaciones que controlen la conducta y determinen el aprendizaje explícito, es necesario estudiar la existencia de las actitudes implícitas determinadas por la identificación de restos (no introspectivamente identificados) de la experiencia pasada que median favorablemente $o$ desfavorablemente sentimientos, pensamientos o acciones hacia objetos sociales. ${ }^{27}$

En el caso de las personas que estudian o ejercen la medicina, es importante evaluar las actitudes implícitas. La existencia de actitudes implícitas negativas relacionadas con el género, el peso, la edad, etc., constituye una limitación para la adquisición de conocimientos y habilidades académicas, y también para desarrollar la empatía necesaria a la hora de brindar asistencia al activarse el sesgo inconsciente.

Durante la atención clínica, cuando los recursos cognitivos son desafiados por las limitaciones del tiempo o del estrés, puede activarse de forma automática e inconsciente el sesgo y provocar que el médico etiquete a un paciente como un miembro de un intergrupo o un intragrupo, y le aplique caracterizaciones estereotipadas positivas o negativas. ${ }^{28}$

El conocimiento previo de estas actitudes inconscientes permitirá que durante la planificación de los contenidos de las materias de la carrera de medicina sean contempladas y se le otorguen al estudiante las herramientas que 
le permitan desenvolverse mejor con el paciente y atender sus necesidades de la manera más adecuada posible.

\section{BIBLIOGRAFÍA}

1. Reingold E, Ray C. Implicit Cognition. Encyclopedia of cognitive sciences; 2006.

2. Schacter DL. Implicit expressions of memory in organic amnesia: learning of new facts and associations. Human Neurobiol 1987;6(2):107-18.

3. Marr D. Vision: A computational investigation into the humam representation and processing of visual information. MIT Press; 2010.

4. PozoJ. Buscandoa Hall desesperadamente: de la psicología cognitiva a la psicología del conocimiento. Anuario psicología 2003;1(34):3-28.

5. Rozo J, Pérez-Costa AM. Condicionamiento clásico y cognición implícita. Acta Colombiana de Psicología 2006;1(9):63-75.

6. Leyens J, Paladino P, R. R-T, Vaes J, DemoulinS, RodriguezPérez A. The emotional side of prejuice: the attribution of secondary emotions to ingroups and outgroups. Pers Soc Psychol Rev 2000(4):186-97.

7. Dorantes Argandar G. Project implicit y el implicit Association Test. Con Sciencia 2010;7(13):13-20.

8. Kandel E, Hawkins R. The biological basis of learning and individuality. Scientific American 1992;267(3):78-86.

9. Baron Cohen S. Mindblindness: An essay on autism and theory of mind. Cambridge: MIT Press; 1997.

10. Leslie A. Pretense and representation: the origins of "theory of mind". Psychol Rev 1987;94:412-42.

11. McGrayne S. The theory that would not die: how Bayes' rule cracked the enigma code, hunted down russian submarines, and emerged triumphant from two centuries of controversy. Published online: 25 junio 2012. DOI: 10.1111/j.1745-3984.2012.00171.x

12. Jacobs R, Kruschke J. Bayesian learning theory applied to human cognition. Cognitive Science 2011; 2 (1):8-21.

13. Estrada C, Oyarzún M, Yzerbyt V. Teorías implícitas y Esencialismo psicológico: Herramientas conceptuales para el estudio de las relaciones entre y dentro de los grupos. Psykhe 2007;16(1):111-21.

14. Levi S, Chi-yue C, Ying-yi H. Lay theories ant intergroup relations. Groups processes and intergroup relations. Relations 2006;21(9):5-24.

15. Haslam N, Rothschild L, Ernst D. Essentialist beliefs about social categories. Brit J Soc Psychol 2000(39):113-27.

16. Yzerbyt $V$, CorneilleO,EstradaC. Theinterplay of subjective essentialism and entitavity in the formation of stereotypes. Personal Soc Psychol Rev 2001;5:141-55.

17. Medin D. Concepts and conceptual structure. Am Psychol 1989(44):1469-81.

18. Estrada Goic CA. El impacto del esencialismo psicológico sobre la percepción de la disidencia intragrupal. Interam J Psychol 2010;44(2):352-60.

19. Estrada C, Yzerbyt V, Seron E. El efecto del esencialismo psicológico sobre las teorías ingenuas de las diferencias grupales. Psicotherma 2004;16(2):181-6.

20. Bar-Tal D. Causes and consequences of delegitimization: Models of conflict and ethnocentrism. J Soc Iss 1990(46):6581.

21. Banaji M, Bhaskar R. Implicit stereotypes and memory: the bounded rationality of social beliefs. Harvard University Press; 2000.

22. Banaji M. Social psychology of stereotypes. International encyclopedia of the social and behavioral sciences; 2001.

23. More D, Loewenstein G. Self-interest, automaticity and the psychology of conflict interest. Soc Just Res 2004;2(17):189202.

24. Smith E. Mental representation and memory. Thehandbook of social psychology; 1998.

25. Banaji M, Greenwald AG, Nosek B. Project Implicit. 1998; Available from: https:/ /implicit.harvard.edu/implicit.

26. Dunham Y, Baron A, Banaji M. From american city to japanese village: the omnipresent of implicit association test. Child Develop 2006;77:1268-81.

27. Greenwald A, Banaji M. Implicit social cognition: attitudes, self-esteem, and stereotypes. Psychol Rev 1995;102(1):4-27.

28. TealC, Gill A, Green A. Helping medical learners recognise and manage unconscious bias toward certain patient groups. Med Educ 2012;46(1):80-8. 